\title{
STARE velocities: 2. Evening westward electron flow
}

\author{
M. Uspensky ${ }^{1,5}$, A. Koustov ${ }^{2}$, P. Janhunen ${ }^{1}$, E. Nielsen ${ }^{3}$, K. Kauristie ${ }^{1}$, O. Amm ${ }^{1}$, R. Pellinen ${ }^{1}$, H. Opgenoorth ${ }^{4}$, and \\ R. Pirjola ${ }^{1}$ \\ ${ }^{1}$ Finnish Meteorological Institute, Geophysical Research, P.O.Box 503, FIN-00101, Helsinki, Finland \\ ${ }^{2}$ Institute of Space and Atmospheric Studies, University of Saskatchewan, Saskatoon, S7N 5E2, Canada \\ ${ }^{3}$ Max-Planck-Institute für Aeronomie, D-37191 Katlenburg-Lindau, Germany \\ ${ }^{4}$ Swedish Institute of Space Physics, Box 537, SE-751 21, Uppsala, Sweden \\ ${ }^{5}$ On leave from Murmansk State Technical University, Sportivnaya 13, Murmansk, 183010, Russia
}

Received: 14 February 2003 - Revised: 27 August 2003 - Accepted: 4 September 2003 - Published: 2 April 2004

\begin{abstract}
Four evening events and one morning event of joint EISCAT/STARE observations during $\sim 22 \mathrm{~h}$ are considered and the differences between observed STARE line-ofsight (1-o-s) velocities and EISCAT electron drift velocities projected onto the STARE beams are studied. We demonstrate that the double-pulse technique, which is currently in use in the STARE routine data handling, typically underestimates the true phase velocity as inferred from the multi-pulse STARE data. We show that the STARE velocities are persistently smaller (1.5-2 times) than the EISCAT velocities, even for the multi-pulse data. The effect seems to be more pronounced in the evening sector when the Finland radar observes at large flow angles. We evaluate the performance of the ion-acoustic approach (IAA, Nielsen and Schlegel, 1985) and the off-orthogonal fluid approach (OOFA, Uspensky et al., 2003) techniques to predict the true electron drift velocity for the base event of 12 February 1999. The IAA technique predicts the convection reasonably well for enhanced flows of $>\sim 1000 \mathrm{~m} / \mathrm{s}$, but not so well for slower ones. By considering the EISCAT $N(h)$ profiles, we derive the effective aspect angle and effective altitude of backscatter, and use this information for application of the OOFA technique. We demonstrate that the OOFA predictions for the base event are superior over the IAA predictions and thus, we confirm that OOFA predicts the electron velocities reasonably well in the evening sector, in addition to the morning sector, as concluded by Uspensky et al. (2003). To check how "robust" the OOFA model is and how successful it is for convection estimates without the EISCAT support, we analysed three additional evening events and one additional morning event for which information on $N(h)$ profiles was intentionally ignored. By accepting the mean STARE/EISCAT velocity ratio of 0.55 and the mean azimuth rotation of $9^{\circ}$ (derived for the basic event), we show that the OOFA performs reasonably well for these additional events.
\end{abstract}

Key words. Ionosphere (ionospheric irregularities, plasma waves and instabilities, auroral ionosphere)

Correspondence to: $\mathrm{M}$. Uspensky

(mikhail.uspensky@fmi.fi)

\section{Introduction}

The STARE coherent radars (STARE: Scandinavian Twin Auroral Radar Experiment) are a useful instrument for convection mapping and related studies (Greenwald et al., 1978; Inhester et al., 1981; Baumjohann and Opgenoorth, 1984; Nielsen, 1989; Nielsen et al., 1999; Kosch and Nielsen, 2001; Nielsen and Rietveld, 2003). In the standard mode, the ionospheric (convection) electron drift magnitude and direction are obtained by stereoscopic merging of two line-ofsight (1-o-s) velocities at every beam crossing. According to Nielsen and Schlegel (1985) the stereoscopic STARE convection estimates are reliable for ionospheric conditions with relatively slow total flows of less than $\sim 500 \mathrm{~m} / \mathrm{s}$ and l-o-s velocities less than $\sim 300$ and $\sim 400 \mathrm{~m} / \mathrm{s}$ in westward and eastward electron flows, respectively. For faster flows the radars were found to underestimate the convection magnitude because the observed velocity along the flow is not the component of the $\boldsymbol{E} \times \boldsymbol{B}$ electron drift (as assumed in the merge approach) but something which is close to the ion-acoustic speed of the medium. This property was attributed to nonlinear effects in the course of the Farley-Buneman (FB) plasma instability development. Nielsen and Schlegel (1985) were able to experimentally establish the 1-o-s velocity-electron flow velocity relationship for these directions, and this work allowed them to modify the original data reduction so that a new method, termed the ion-acoustic approach (IAA), was developed, to successfully infer plasma convection from the original l-o-s velocities.

It is well known that the electrojet irregularities can be either Type 1 or Type 2 (Fejer and Kelley, 1980). Type 1 irregularities are quite strong plasma fluctuations excited along the electron flow within limited cones of aspect (offorthogonal) and flow angles (within a cone limited by \pm 1 and $\pm 30^{\circ}$ sectors, respectively), where the linear instability growth rate is positive (in-cone irregularities). These irregularities are only excited when the plasma drift exceeds the Farley-Buneman instability threshold of $300-400 \mathrm{~m} / \mathrm{s}$ (the ion-acoustic speed at the E-region heights). It is generally accepted that Type 1 irregularities move approximately with 
the ion-acoustic speed. If so, then one cannot directly use Doppler measurements from such directions for the stereoscopic derivation of plasma convection. Type 2 irregularities are relatively weak plasma fluctuations that can be seen at large flow angles and/or at increased off-orthogonal angles (out-of-cone irregularities), and it is widely accepted that their velocity is close to the "cosine" component of the $\boldsymbol{V}_{E \times B}$ electron drift along the radar beam. For the STARE experiment, the Norway radar quite often sees Type 1 irregularities, while the Finland radar sees typically Type 2 irregularities, since the former radar observes close to the L-shell directions, while the latter one observes perpendicular to the L-shell directions.

Both cosine merge and IAA techniques (Nielsen et al., 2002) assume that for observations at large flow angles (i.e. outside the FB instability cone), the irregularity phase velocity is a cosine component of the $\boldsymbol{E} \times \boldsymbol{B}$ plasma drift along the radar beam, as predicted by the linear fluid theory of the FB instability (Fejer and Kelley, 1980). This property is instrumental for the success of the STARE convection predictions in most cases, as one can infer from the results of STARE/EISCAT comparisons performed by Nielsen and Schlegel (1985). More detailed scrutiny of STARE/EISCAT data, however, shows some inconsistencies (Kustov et al., 1990; Kustov and Haldoupis, 1992; Koustov et al., 2002; Uspensky et al., 2003).

In late 1997 the STARE radars were upgraded so that a new double pulse separation of $200 \mu$ s was adopted (as compared to the original separation of $300 \mu \mathrm{s}$ ) and a multi-pulse transmission, in addition to the single-to-double-pulse sequence (Greenwald et al., 1978), was added. In spite of years of operation, the assessment work on the performance of the new system has just begun. STARE/EISCAT comparisons by Koustov et al. (2002) and Uspensky et al. (2003) discovered significant and systematic differences between STARE convection estimates (standard double-pulse mode) and the EISCAT electron flow measurements. Koustov et al. (2002) considered evening sector STARE/EISCAT observations and showed that the Finland STARE velocities were $\sim 2$ times smaller than the EISCAT $\boldsymbol{E} \times \boldsymbol{B}$ drifts along the radar beam. These authors explained the effect by the non-orthogonality of backscatter. Uspensky et al. (2003) hereafter referred to as paper 1, considered morning sector STARE/EISCAT measurements and discovered that there were periods when the Finland radar velocity was actually larger than the EISCAT $\boldsymbol{E} \times \boldsymbol{B}$ electron flow component. These authors argued that besides non-orthogonality of scatter, ion motions at high altitudes can contribute significantly to the observed velocity. It was pointed out that the cosine rule for the velocity can be violated because of the above effect. These two papers are, in some sense, in line with a recent study by Nielsen et al. (2002), who explored older (late 1980s) STARE/EISCAT (ERRIS) observations and demonstrated a more complicated relationship between the STARE 1-o-s velocity, the ion-acoustic speed and the matched 1-o-s EISCAT electron flow velocity component ("matched" means the EISCAT velocity projected to the STARE line-of-sight).
All three studies provide a basis for further investigations of the relationship between the VHF Doppler velocity and the $\boldsymbol{E} \times \boldsymbol{B}$ plasma drift.

There is a practical aspect to the issue. If the phase velocity of plasma waves at large flow angles is not very close to the convection velocity component, then how reliable are the IAA convection estimates? Paper 1 investigated the question for the morning sector observations and showed that the IAA method still works reasonably well. The authors also found that comparably good convection estimates can be achieved by a simple stereoscopic merging of the corrected l-o-s velocities, if one takes into account the fundamental fact that an auroral radar never "sees" pure orthogonal backscatter. This is applicable to both the Norway and Finland radars which collect echoes on the EISCAT flux tube, although it is well known that pure orthogonality is satisfied at $\sim 100 \mathrm{~km}$. This new method of convection estimate was termed the offorthogonal fluid approach (OOFA). The overall performance of the OOFA method for other events has not been assessed yet.

For the evening sector observations of Koustov et al. (2002), the situation seemed more difficult to deal with. In this case, both the Finland and Norway STARE radars were observing mainly outside the FB instability cone (estimates were based on simultaneous EISCAT measurements of the plasma drift and ion-acoustic speed), and both radars had velocites well below the $\boldsymbol{E} \times \boldsymbol{B}$ component. Koustov et al. (2002) showed that the standard stereoscopic technique underestimates convection for the event considered. Convection predictions according to the IAA and OOFA methods were not explored.

The present paper compares STARE Doppler velocities and EISCAT electron drift velocities in the evening sector and evaluates the performance of the IAA and OOFA techniques. The difference from the previous studies is that we use multi-pulse STARE data. First, the efforts are concentrated on the event considered by Koustov et al. (2002), because information on the electron density profiles is available for this event. Analysis of this event also allows one to "calibrate" the OOFA convection estimates against EISCAT measurements and thus, to give information on the STARE velocity depression coefficient in the evening sector suitable for the analysis of other events. The calibration procedure considered in this study is a combination of methods used by Kustov et al. (1990) and in paper 1.

Then, three new evening sector events are considered. For these test events no information on the electron density distribution was used, so that they are representative for an assessment of OOFA performance. We also consider, for a comparison, the morning sector event studied in paper 1.

\section{Stereoscopic merging and off-orthogonality approach}

As mentioned in the previous section, in the original STARE method, it was assumed that the phase velocity of $\sim 1$-m irregularities along a specific radar beam is the cosine 
component of the plasma $\boldsymbol{V}_{\boldsymbol{E} \times \boldsymbol{B}}$ drift (Greenwald et al., 1978). Kustov et al. (1990) and Koustov et al. (2002) noted that the deficiency of this assumption is in adopting pure orthogonality of backscatter. Paper 1 went even further by declaring that the auroral backscatter is always effectively non-orthogonal in a sense that for any radar cell, the auroral echo is collected from various heights, at which a perfect orthogonality exists at only one height. These authors considered finer features in the irregularity velocity variation with height (as compared to the previous study by Kustov et al., 1990) and introduced the OOFA name for such a consideration. The OOFA concept is the main idea that we investigate further in the present study.

Let us quickly recall the main points of the OOFA method. Fundamentally, it is postulated that for the out-of-cone irregularities, the linear fluid theory equation describes properly the irregularity phase velocity $\boldsymbol{V}_{p h}$ (Fejer and Kelley, 1980),

$\boldsymbol{V}_{p h}=\left(\boldsymbol{V}_{\boldsymbol{e}}+R \boldsymbol{V}_{\boldsymbol{i}}\right) /(1+R)$.

Here, $R=R_{o}\left(\cos ^{2} \Psi+\left(\Omega_{e}^{2} / v_{e}^{2}\right) \sin ^{2} \Psi\right)$ and $R_{o}=v_{e} v_{i} / \Omega_{e} \Omega_{i}$, where $v_{e, i}$ and $\Omega_{e, i}$ are the electron and ion collision frequencies with neutrals and the gyrofrequencies, respectively, $\boldsymbol{V}_{e, i}$ are the electron and ion drift velocities, and $\Psi$ is the off-orthogonal (or aspect) angle. Since a coherent radar measures the component of this velocity, $V_{p h}^{(k)}$, along a specific beam direction, the 1-o-s velocity is

$V_{p h}^{(\boldsymbol{k})}=(\boldsymbol{k} /|k|) \cdot\left(\boldsymbol{V}_{\boldsymbol{e}}+R \boldsymbol{V}_{\boldsymbol{i}}\right) /(1+R)$.

It is assumed that any ionospheric echo is received from a "thick" layer (e.g. 95-125 km) of irregularities and the contribution from each height is defined by the local electron density and the aspect angle of the waves (Uspensky, 1985). To characterize these effects, the authors of paper 1 proposed to consider the aspect angle, the electron density and the backscatter height as power normalised effective quantities:

$\Psi_{e f f}=\int P(h)|\Psi(h)| d h / \int P(h) d h$,

$N_{e f f}=\left(\int P(h) N^{2}(h) d h / \int P(h) d h\right)^{1 / 2}$,

$h_{e f f}=\int P(h) h d h / \int P(h) d h$.

Under specific conditions this should be done for each radar of the pair separately. In these equations, $P(h)$ is the relative backscatter power at the specific height at which the local aspect angle $\Psi(h)$ assumes a certain value, $P(h) \propto$ $<(\delta N / N)^{2}>\left(N(h) / N_{\max }\right)^{2} \exp \left(-a^{2} \tan ^{2} \Psi(h)\right)$. The fractional electron density fluctuation amplitude $\left\langle(\delta N / N)>^{1 / 2}\right.$ is assumed to be height-independent (for simplicity) as rocket measurements show, for example as in Pfaff et al. (1984). The parameter $a$ defining the strength of the power attenuation with the aspect angle is assumed to be $a \sim 50$ (such a value gives the mean power attenuation of $\sim 10 \mathrm{~dB} /{ }^{\circ}$ for the aspect angle interval of $0-3^{\circ}$ ) that is slightly

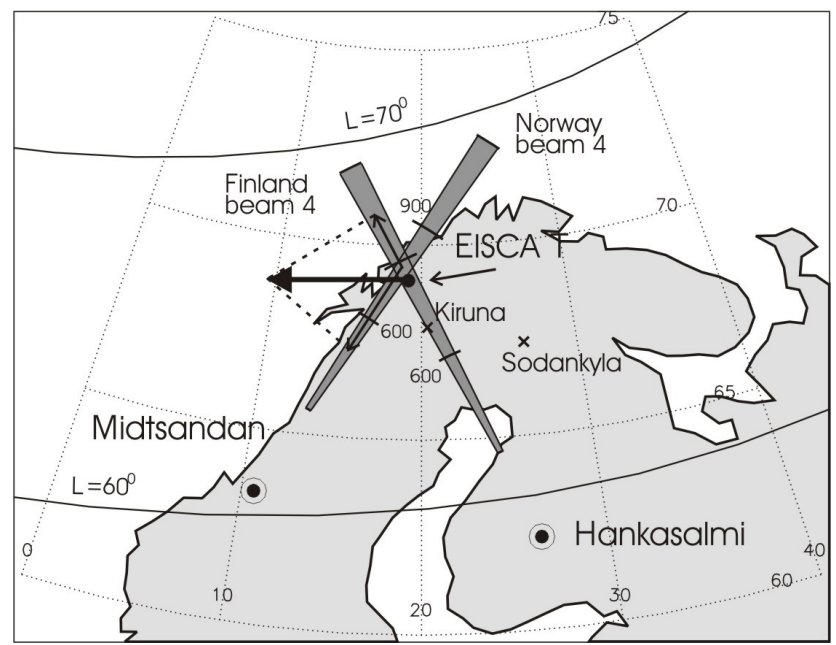

Fig. 1. Field of view of the Hankasalmi Finland STARE radar beam 4, and the Midtsandan Norway STARE radar beam 4 assuming $110 \mathrm{~km}$ height of scatter. Lines across the beams are slant range marks of 600 and $900 \mathrm{~km}$. The STARE irregularity drift velocity vector is the cosine-merged product of the two measured velocities. The solid dot denotes the area where ionospheric parameters were measured by the EISCAT incoherent scatter facility, which includes a UHF transmitter/receiver in Troms $\varnothing$ and receivers in Kiruna and Sodankylä (crosses). The solid thick lines represent the PACE magnetic latitudes.

larger than the one used by Farley et al. (1981) for the equatorial electrojet.

Paper 1 adopted the linear variation of the aspect angle with height, with a mean gradients of $0.075^{\circ} / \mathrm{km}$ (for the sake of simplicity), although the real Finland and Norway height gradients in the EISCAT flux tube are $\sim 0.07$ and $\sim 0.08^{\circ} / \mathrm{km}$, respectively (see paper 1 ). The height of zero aspect angle was assumed to be $100 \mathrm{~km}$ for both radars, although those are $\sim 97$ and $\sim 99 \mathrm{~km}$ for the Finland and Norway radars, respectively (Koustov et al., 2002). The reason is that inside the radar collected area of $\sim 15 \times 50 \mathrm{~km}^{2}$, the height is varied by a few $\mathrm{km}$ up and down with the higher altitudes being more effective for a stronger backscatter.

The effective values of $\Psi_{\text {eff }}$ and $h_{\text {eff }}$ obtained from Eqs. (2) and (4) give estimates for the aspect angle and height needed to solve Eq. (1) quantitatively. One can define the ion contribution in the irregularity phase velocity as the clockwise rotation of the vector $\boldsymbol{V}_{p h}$ with respect to the vector $\boldsymbol{V}_{\boldsymbol{e}}$ as well as find the ratio of $\boldsymbol{V}_{p h}$ and $\boldsymbol{V}_{\boldsymbol{e}}$ magnitudes. We stress that the above estimates can only be done if the electron density profiles in the backscatter volume (here in the EISCAT flux tube) are known. A fair question is what are the typical values of $\Psi_{e f f}$ and $h_{e f f}$, and whether those found for one individual event can be used for the analysis of other events. More work is needed to answer this question. Comparisons performed in this study indicate that the estimates obtained for the clockwise azimuth rotation of the irregularity phase velocity vector and the ratio of the irregularity phase velocity to the electron flow velocity (the so-called velocity depression coefficient) are reasonable. 


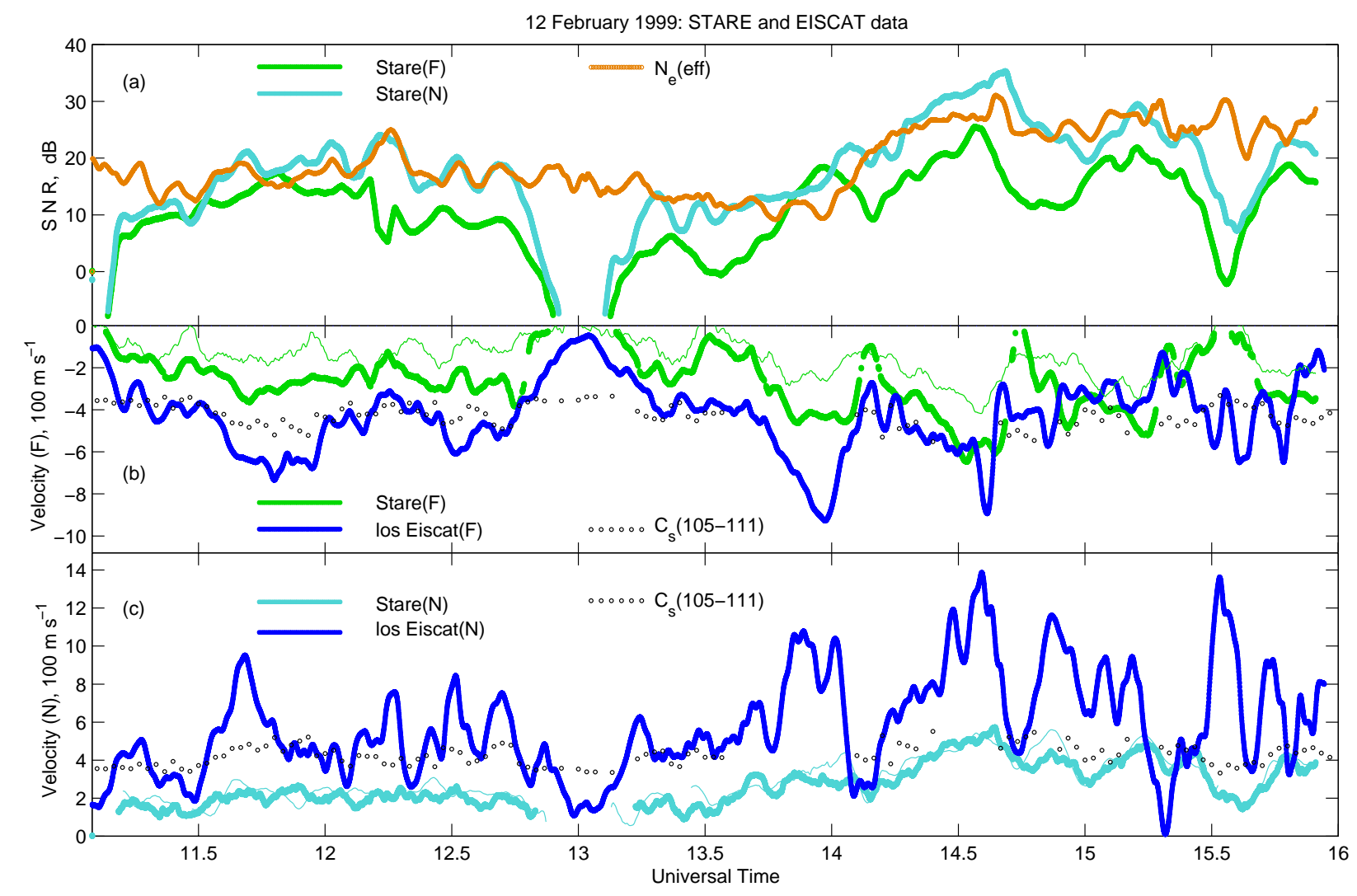

Fig. 2. The STARE (Finland radar beam 4 and the Norway radar beam 4) and EISCAT parameters: (a) SNR, green line for Finland and light blue line for Norway; the orange line is the effective electron density (on a logarithmic scale); (b) Finland beam 4 line-of-sight MP and DP velocities, thick and thin green line, respectively, and matched l-o-s EISCAT $\boldsymbol{V}_{\boldsymbol{E} \times \boldsymbol{B}}$ velocity component, dark blue line; open black circles in panels (b) and (c) are the isothermal ion-acoustic speed in height interval of $105-111 \mathrm{~km}$ according to EISCAT; (c) the same as in (b) but for the STARE-Norway beam 4 l-o-s MP and DP velocity, thin and thick light blue line (opposite marking), and matched l-o-s EISCAT $\boldsymbol{V}_{\boldsymbol{E} \times \boldsymbol{B}}$ velocity component, dark blue line, respectively.

\section{$3 \quad 12$ February 1999 event}

We start by considering joint STARE/EISCAT data between 11:00 and 16:00 UT on 12 February 1999, which were partly described by Koustov et al. (2002). We consider comparison at one point, similarly to Nielsen and Schlegel (1985), Koustov et al. (2002) and paper 1. This point is located on flux lines close to the EISCAT spot that corresponds to Finland radar beam 4 (note the recent changes at http://www.geo. fmi.fi/PLASMA/RADAR/STARE), range $870 \mathrm{~km}$ and Norway beam 4, range $775 \mathrm{~km}$, shown in Fig. 1. Note that for the area of measurements, MLT $\sim \mathrm{UT}+2$. The STARE velocity and power were measured using the single-to-double and multiple pulse pattern with 20-s averaging. The EISCAT UHF radar was run in the standard tri-static mode CP-1K of plasma flow measurements, with the Troms $\varnothing$ antenna being pointed along the local magnetic field line and the Kiruna and Sodankylä receiver beams being oriented toward a common volume at a height of $\sim 250 \mathrm{~km}$. EISCAT provided information on the electric field, electron density and electron and ion temperatures in both E- and F-regions. The altitude resolution of the density and temperature measurements was $\sim 3.1 \mathrm{~km}$ below $\sim 180 \mathrm{~km}$ and $\sim 22 \mathrm{~km}$ above $\sim 180 \mathrm{~km}$, and the time resolution was $1 \mathrm{~min}$. The diameter of the EISCAT beam spot is $\sim 1 \mathrm{~km}$ in the E-layer and $\sim 2.6 \mathrm{~km}$ in the Flayer, meaning that the E-layer (F-layer) horizontal projection of the EISCAT scattering volume has an area of about 3 orders ( 2 orders) of magnitude smaller than the collecting areas of the STARE radars. In our presentation we adopted common 4-min averaging for all data, to smooth out noiselike velocity variations known for the event (Koustov et al., 2002).

The afternoon and the early evening of 12 February 1999 were a moderately disturbed period. The positive magnetic perturbations over Scandinavia detected by the IMAGE magnetometers were 100-150 nT, with two 200-250 nT spikes prior to 14:00 UT and, shortly afterwards, around 14:30 UT. Both STARE radars detected backscatter in a broad band of ranges covering the EISCAT spot, stretching all the way to the E-layer radio horizon.

Figure 2 presents the data for the whole period under study. Panel (a) illustrates the echo signal-to-noise ratios (SNRs) in the STARE Finland radar beam 4, green (azimuth of the EISCAT flux tube is $-28^{\circ}$ ), and the STARE Norway 

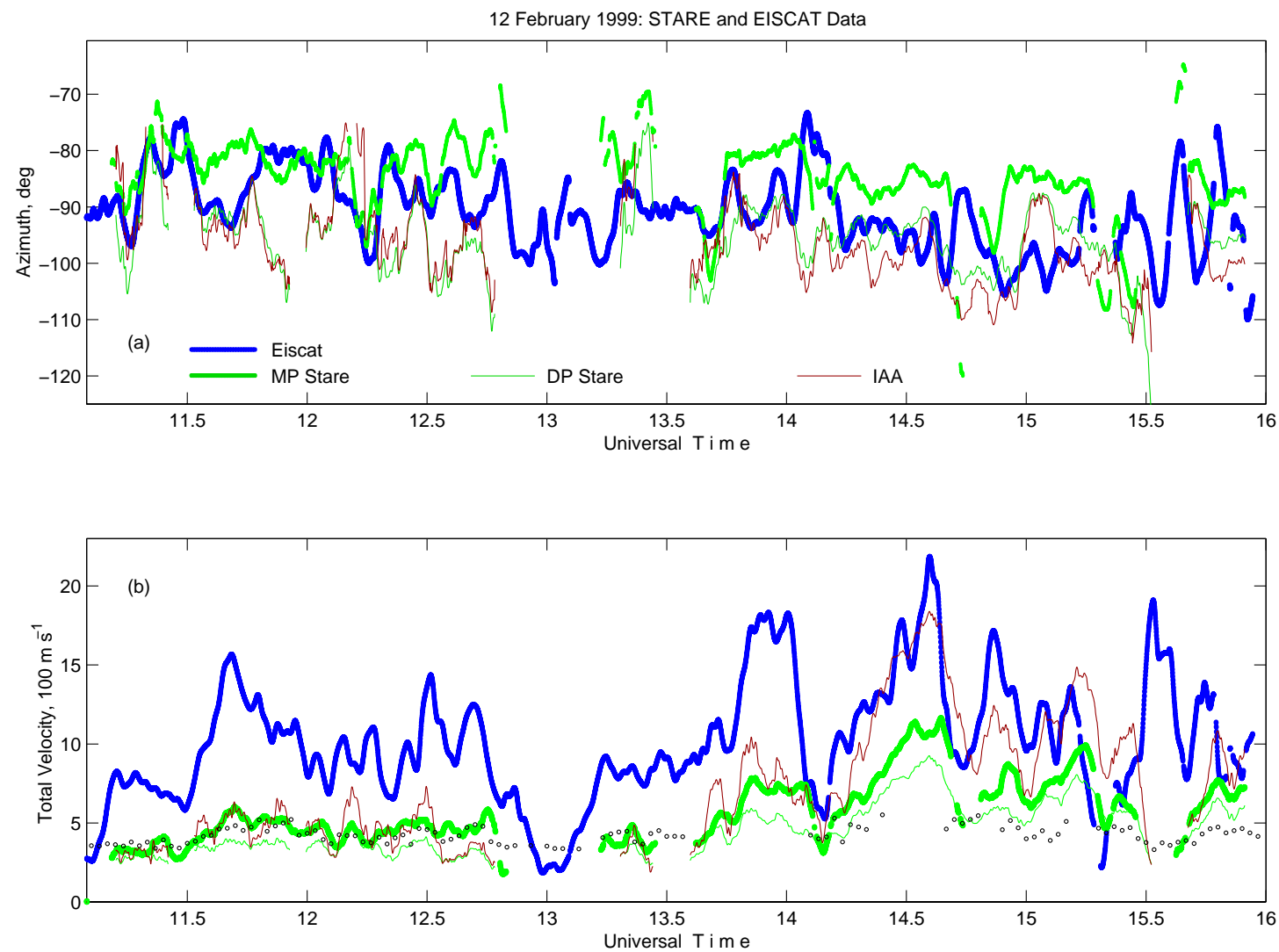

Fig. 3. The STARE and EISCAT parameters matched to Fig. 2 with three data pairs given in panels (a) and (b) of the azimuth and the velocity, respectively: (1) the STARE MP and DP merged flow azimuth and velocity, thick and thin green line, (2) the EISCAT $\boldsymbol{V}_{\boldsymbol{E} \times \boldsymbol{B}}$ electron flow azimuth and velocity, blue line, and (3) the STARE IAA electron velocity azimuth and velocity, brown line; open black circles are the isothermal ion-acoustic speed in the height interval of $105-111 \mathrm{~km}$ according to EISCAT.

radar beam 4, light blue (azimuth $34^{\circ}$ ), respectively. The Norway SNRs were decreased by $\sim 1.5 \mathrm{~dB}$ to account for the difference in the radar distances to the scattering point (assuming a $R^{-3}$ factor of power attenuation). Though it is not the main interest of this study, below we present a concise description of SNR signatures to describe the condition of STARE measurements.

Both the Norway and Finland SNRs exhibited a similar behaviour. (For known beam azimuth we see from Fig. 3a that the flow angles were of $61-51$ and $60-70^{\circ}$, respectively). Due to the larger flow angles, the Finland signal was slightly weaker. The moderate SNR difference can also be a product of the different orientation of the $\sim 15 \times 50 \mathrm{~km}^{2}$ radar collecting cell. In Fig. 2 the orange line exhibits arbitrary adjusted EISCAT-based effective electron density for the Norway STARE SNRs. We present the electron densities on a logarithmic scale adjusted to the values of SNR, so that if the echo power variations were only a result of electron density changes $\left(\mathrm{SNR} \propto N^{2}\right)$, one would see a linear relationship; $20 \mathrm{~dB}$ SNR roughly corresponds to a density of $\sim 0.5 \times 10^{11} \mathrm{~m}^{-3}$ (for more details see Fig. 5a below). A doubling (halving) of electron density would make a 6-dB positive (negative) change in the SNR scale.
There are two prolonged SNR enhancements centred at 12:00 UT and around 14:15-15:30 UT, when the EISCAT electron flow velocities were around $1000 \mathrm{~m} / \mathrm{s}$ or more. The overall SNR increase from the first to the second maximum is $10-12 \mathrm{~dB}$. It agrees well with the effective electron density increase by a factor of $\sim 3$. At 11:30-12:40 UT SNRs show $5-8 \mathrm{~dB} \sim 15$-min pulsations which were similar in their phase and magnitude to pulsations in the effective electron density. A similar correlation of echo power and effective density was described in paper 1 for the morning case. The correlation is not a surprise; it is expected for very fast plasma flows (Oksman et al., 1986; Nielsen et al., 1988) which certainly was the case.

The SNR's variations showed some response to the electric field as well. For example, there were three drops in both the Norway and Finland echo power at $\sim 11: 15,13: 00$ and 15:30 UT, without concurrent significant changes in the electron density. These periods were characterised by significantly decreased electric field magnitudes, according to EISCAT. (see the EISCAT electron drift magnitude in Fig. 3, panel (b)), and strong decreases in the magnetic field X components on nearby IMAGE magnetometers, for which data are not presented here. 
In panels (b) and (c) we show STARE multi-pulse (MP) l-o-s velocities (thick green and thin light blue lines for the Finland beam 4 and Norway beam 4, respectively). We derived them as the mean phase angle slope, $\tan ^{-1}(\mathrm{Im} / \mathrm{Re})$, derived from the real and imaginary parts of the ACF as a function of the lag number (Hanuise et al., 1993), as it is done in the SuperDARN data reduction scheme (Greenwald et al., 1995). Heavy dark blue lines are the 1-o-s EISCAT convection velocity components along each beam. The black open circles in panels (b) and (c) are the "isothermal" ion-acoustic speed $C_{S}$ (Farley and Providakes, 1989), as estimated from the EISCAT temperatures $T_{e}$ and $T_{i}$ in the height interval of $105-111 \mathrm{~km}$, assuming the electron and ion specific heat ratio of 1 . One can see that both Finland and Norway MP velocities are mostly below the ion-acoustic speed in the first half of the time interval and close to it in the second half. The thin green line in panel (b) and thick light-blue line in panel (c) are the Finland and Norway standard double-pulse (DP) velocities. The Finland DP velocity is systematically less in magnitude than the matched Finland MP velocity. In contrast to the Finland data, the Norway DP velocity in panel (c) is reasonably matched to the Norway MP velocity. Thick lines (green in panel (b) and light-blue line in panel (c)) indicate that these are the ones that are used below for the stereoscopic data merging and subsequent OOFA calculations.

The differences between MP and DP velocities and their nature are the subject of a separate study. Here we mention briefly the main features. The Norway DP and MP velocities were close to each other in the evening and morning for the whole set of cases that we considered. The Finland DP and MP velocities were close to each other only in the morning (as in paper 1), and there were significant differences in the evening. For afternoon-evening measurements, the MP velocities were typically larger (by a factor of 1.5-2) than the DP velocities (Fig. 2b, thick and thin green lines, respectively). Overall, the STARE 13-lag autocorrelation functions (ACFs) were noisier for the Norway radar than for the Finland radar; however, for some time intervals they were reasonably good for both systems. The Norway ACFs were good during $\sim 1.5 \mathrm{~h}$ in total, around 12:40, 14:30 and 15:20 UT. For the periods of noisy Norway ACFs, only three lags (1, 3 and 4) could have been used for the MP velocity estimates, because other lags demonstrated very irregular changes from lag to lag. The average Norway MP velocities determined through the phase angle slope of these 3 lags (Hanuise et al., 1993) are presented in panel (c). These simplified velocity estimates agree well with the full ACF-fit velocities for those intervals when the Norway ACFs were good, so that we are confident that the simplified procedure gives a reasonable velocity estimate. Since the Norway DP velocities were very close to the MP velocities and there were some problems in deriving the full MP velocities, we decided to use DP Norway velocities for the convection estimates.

Figure 3 a shows the azimuth of plasma flow according to EISCAT (dark blue lines) and to the STARE "stereoscopic" method. The thick green line shows MP data, and the thin green line shows the DP data. One can see the more clock- wise orientation of the MP merge velocity with respect to the EISCAT azimuth and the more counterclockwise rotation of the DP merge velocity. Panel (b) shows the behaviour of the EISCAT and STARE MP merged velocities (dark blue and thick green lines, respectively, as well as the STARE merged DP velocity (thin line)). Open black circles are (as earlier) the EISCAT isothermal ion-acoustic speed. Brown lines in panels (a) and (b) show the STARE IAA electron flow prediction by Nielsen and Schlegel (1985) based on the STARE DP velocity data. Though the electron flow velocities are rather large, $1000-2000 \mathrm{~m} / \mathrm{s}$, the merged STARE phase velocity (Fig. 3b) as well as the 1-o-s Norway phase velocity (Fig. 2c) does not seem to be related to the E-layer plasma temperature. Below we will discuss two features of the data and the IAA predictions that are persistently different before and after $\sim 13: 30$ UT.

During the period 11:10-13:30 UT, the Norway STARE MP/DP velocity (Fig. 2c), as well as the Finland STARE DP velocity (Fig. 2b), were mainly smaller than the evening critical threshold of $\sim 300 \mathrm{~m} / \mathrm{s}$ for the IAA. The Norway STARE MP/DP velocity was two times smaller than the EISCAT isothermal ion-acoustic speed and roughly two times smaller than the EISCAT 1-o-s velocity. The Finland MP velocities were also smaller than the ion-acoustic speed and the EISCAT 1-o-s component but the differences were moderate. One can conclude that both radars observed backscatter from out-of-cone irregularities (as was found earlier by Koustov et al., 2002). Smaller STARE velocities in comparison with the l-o-s EISCAT components (particularly for the Norway radar) do not support a recent assertion by Nielsen et al. (2002) that "for large flow angles, the Doppler shifts are equal to the component of the electron drift velocity on the line of sight". If one merges the MP or DP velocities, the resultant velocities are around the ion-acoustic speed and nearly half the EISCAT electron velocity of $1000-2000 \mathrm{~m} / \mathrm{s}$. The IAA velocity, obtained from the DP data, is close to the DP merged velocity. Another feature of the IAA velocity underestimation can be seen later at 13:40-15:30 UT, when Nielsen's and Schlegel's $\sim 300 \mathrm{~m} / \mathrm{s}$ threshold suggests the existence of in-cone irregularities for the radars. For the second period, mostly between 14:20-15:30 UT, the IAA velocity is reasonably close to the EISCAT measurements (Fig. 3, panel (b)).

The STARE and EISCAT flow azimuth angles showed reasonable mutual agreement in their temporal variations, though a moderate $5-15^{\circ}$ clockwise offset of the STARE MP azimuths and $10-0^{\circ}$ counterclockwise offset of the STARE DP azimuths are visible (Fig. 3, panel (a)). For the overthreshold velocity the counterclockwise IAA azimuth offset seen at 13:40-15:00 UT is slightly larger than for the plain DP velocity (compare thin green and brown line). The clockwise azimuth offset between the EISCAT velocity and the STARE MP merged velocity vectors is consistent with the idea that the ion velocity contributes to the observed total Doppler velocity, as was found in paper 1.

Figure $3 b$, as a whole, shows that the magnitude of the electron drift, derived through the IAA method, experiences 


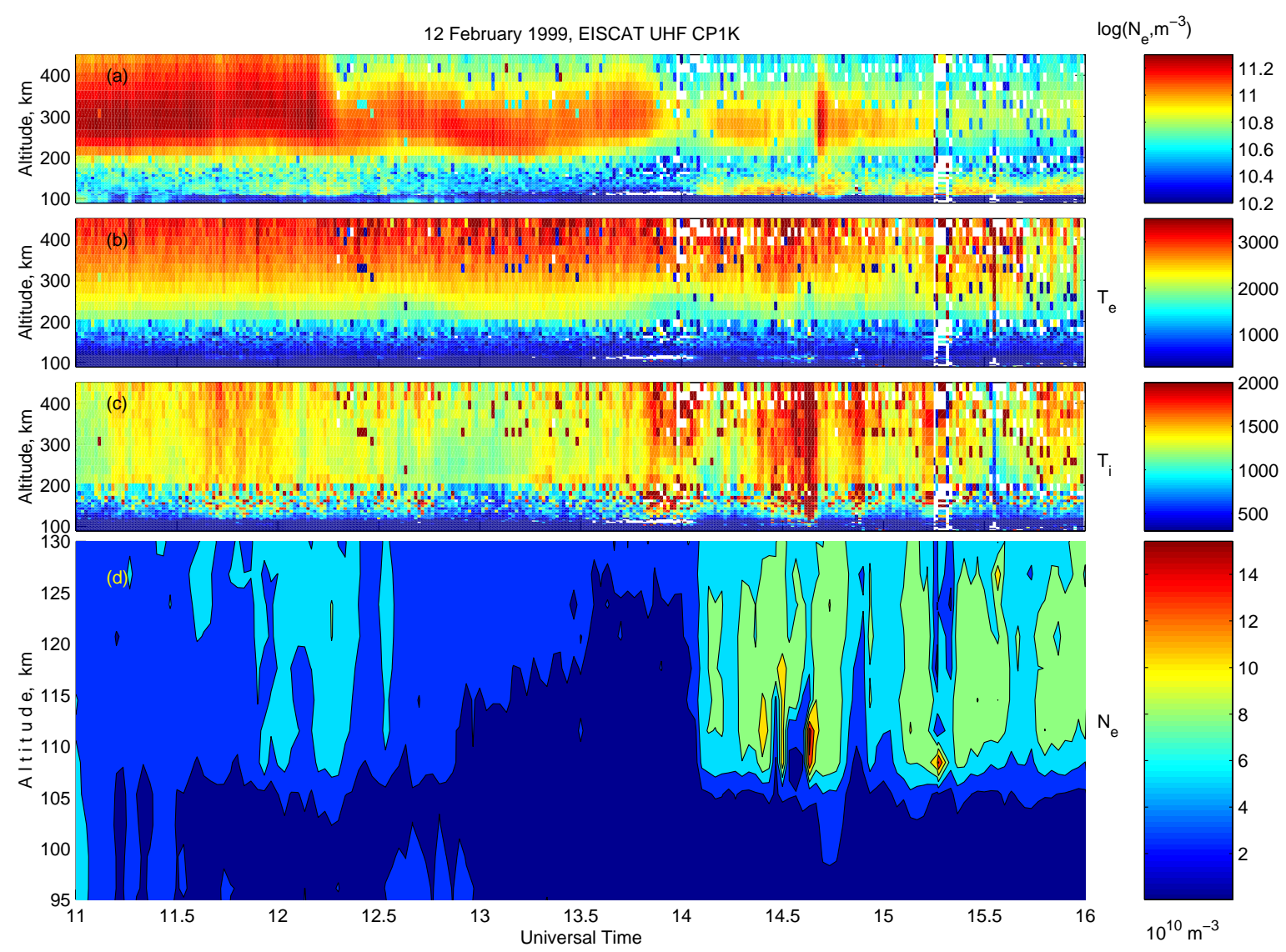

Fig. 4. The EISCAT parameters at 12 February 1999: (a) the E- and F-layer electron density in units of $\log \left(N_{e}, \mathrm{~m}^{-3}\right),(\mathbf{b})$ and (c) the electron and ion temperature, ${ }^{\circ}$, and (d) the E-layer electron density in units of $10^{10} \mathrm{~m}^{-3}$.

significant underestimation compared to the EISCAT electron flow velocity during $60-70 \%$ of the time. This happens when the electron flow is close to or less than $\sim 1000 \mathrm{~m} / \mathrm{s}$. The IAA magnitudes agree reasonably well with the EISCAT values for the second half of the period. We will address this issue later.

The EISCAT parameters for the entire ionosphere and the electron density distribution in the E-layer with better resolution are shown in Fig. 4. A distinct feature here is the two intervals with quite different E-layer density distributions before and after 14:00 UT (panel (d)). Before 14:00 UT, the E-layer was unstructured and moderate-to-low in terms of the density magnitude. During this period, a stable westward electron flow of $\sim 1000 \mathrm{~m} / \mathrm{s}$ (Fig. 3) and positive magnetic perturbations of $\sim 40-50 \mathrm{nT}$ (X-component of the TRO magnetometer, data are not shown here) were observed. An exception is the 10-15 min interval centred at 13:00 UT when the electron velocity quickly dropped down to a few hundred $\mathrm{m} / \mathrm{s}$ and the STARE backscatter disappeared. For the second time interval, after 14:00 UT, the density was enhanced in the upper part of the E-layer and there were seen short-time variations, perhaps in response to auroral particle precipitation with a variable particle flux and energy. At $\sim 14: 40$ and $\sim 15: 20$ UT the TRO magnetic X component shows two short-lived positive spikes overshooting a positive background. The magnetic spikes are well correlated with two STARE power enhancements and a common enhancement of plasma convection (the largest velocities were up to $2000 \mathrm{~m} / \mathrm{s}$, Fig. 2a and Fig. 3 b). More details as to how the STARE echoes react on variations of the ionosphere parameters are presented in the discussion.

\section{OOFA modelling for the 12 February 1999 event}

The EISCAT data for the 12 February 1999 event provide a good opportunity for the assessment and calibration of the OOFA predictions of the plasma convection. Here we first attempt to predict temporal variations of the effective parameters for both STARE radars, Eqs. (2)-(4). Our ultimate goal in these calculations is to determine the typical ratio between the EISCAT and STARE velocities $\left|\boldsymbol{V}_{p h}\right| /\left|\boldsymbol{V}_{\boldsymbol{E} \times \boldsymbol{B}}\right|$ and the typical azimuth turn of the irregularity phase velocity vector $\boldsymbol{V}_{p h}$ with respect to the electron drift vector $\boldsymbol{V}_{\boldsymbol{E} \times \boldsymbol{B}}$.

Figures 5a-c shows the calculated magnitudes of $N_{e f f}$, $h_{\text {eff }}$ and $\Psi_{\text {eff }}$ based on EISCAT electron density profiles between 95 and $125 \mathrm{~km}$. According to Fig. $5 \mathrm{c}$, the effective aspect angle was mainly between 0.7 and $0.9^{\circ}$, reaching maximum values of $\sim 1.1^{\circ}$ for a short period of time around 


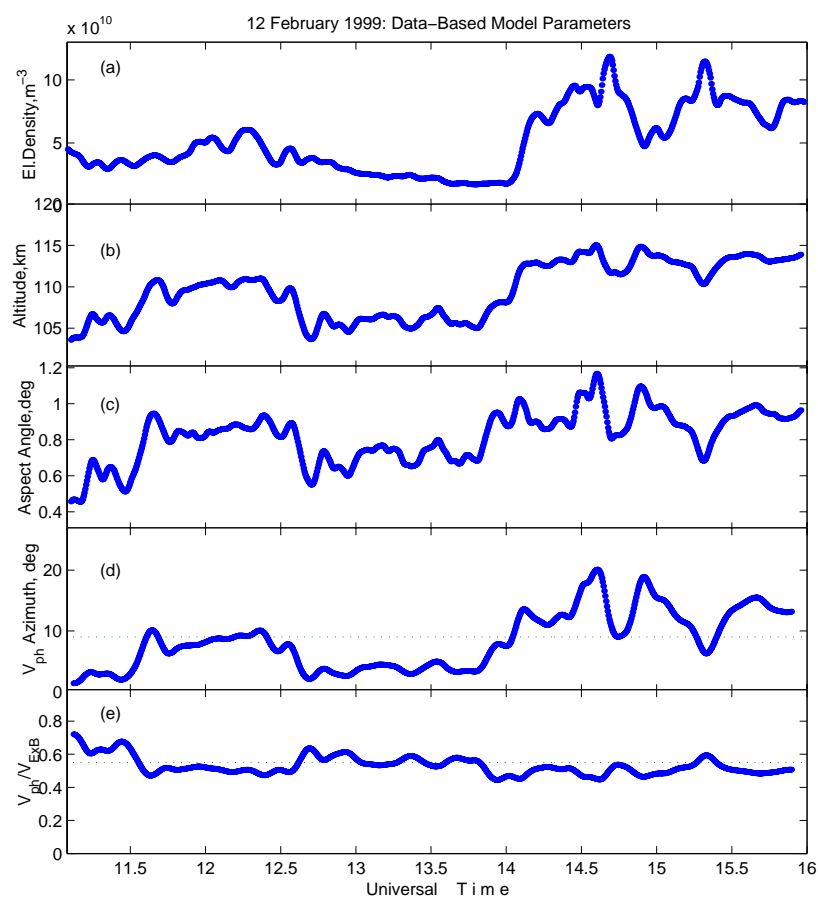

Fig. 5. The OOFA modelling: (a) the effective electron density seen by a radar, (b) the effective backscatter altitude, (c) the effective aspect angle, (d) the clockwise azimuth offset of the largest irregularity phase velocity $\boldsymbol{V}_{p h}$ with respect to the $\boldsymbol{E} \times \boldsymbol{B}$ azimuth and (e) the predicted $\left|\boldsymbol{V}_{p h}\right| /|\boldsymbol{E} \times \boldsymbol{B}|$ velocity ratio. Dotted lines in panels (d) and (e) are their eye-adjusted expected values.

14:30 UT. We stress the fact that the effective aspect angles, as seen by the radar, are not zero, in spite of the perfect orthogonality at the height of $100 \mathrm{~km}$. Still, the magnitudes of $\Psi_{\text {eff }}$ indicate whether observations are being performed inside the FB instability cone in aspect angle. The effective backscatter altitude $h_{\text {eff }}$ was $110-114 \mathrm{~km}$ (see Fig. 5b). The exception was at 12:40-13:50 UT when the E-layer was less dense in its upper part. By comparing Fig. 4 and Figs. 5a-c one can see that the larger effective altitude and aspect angle correspond to a smaller electron density in the bottom $\mathrm{E}$ region and increased density in its upper part.

For the modelling, we assume that the backscatter altitude and aspect angle in Eq. (2) varied with time, as shown in Figs. 5b and c. We use the semi-empirical model for ionneutral collision frequencies of Huuskonen (1989). Electron collision frequencies were computed using the approach by Schlegel (1983).

Panel (d) presents calculations for the turn in the azimuth of $\mathbf{V}_{p h}$ with respect to $\boldsymbol{E} \times \boldsymbol{B}$, and panel (e) shows the ratio $\left|\boldsymbol{V}_{p h}\right| /\left|\boldsymbol{V}_{\boldsymbol{E} \times \boldsymbol{B}}\right|$. One can notice a clockwise $2-20^{\circ}$ rotation of $\mathbf{V}_{p h}$. This rotation is due to the ion motion contribution to the irregularity phase velocity, as prescribed by Eq. (2) and as discussed in paper 1. Apparently, increases in both the backscatter altitude and the aspect angle lead to a larger $\mathbf{V}_{p h}$ azimuth rotation.
The velocity ratio $\left|\boldsymbol{V}_{p h}\right| /\left|\boldsymbol{V}_{\boldsymbol{E} \times \boldsymbol{B}}\right|$, which we call the STARE velocity depression coefficient, is around 0.5-0.6, noticeably smaller than 1 . The ratio does not change considerably, contrary to variations of other parameters shown in Fig. 4. As mentioned in paper 1, this happens because there is a partial compensation of variations due to $|R|$ in the nominator and denominator of Eq. (1). However, there is a tendency for the $\left|\boldsymbol{V}_{p h}\right| /\left|\boldsymbol{V}_{\boldsymbol{E} \times \boldsymbol{B}}\right|$ ratio to be smaller for the larger effective altitudes and aspect angles.

In Fig. 2a we have already reported the effective electron density seen by the Norway radar (arbitrary adjusted orange line). We should note that the above effective density was adjusted for variations of the flow angle by assuming that log (power) $\propto-2 \sin ^{2}\left(\Theta_{N}\right)$, where $\Theta_{N}$ is the flow angle for the Norway radar (Andre, 1983). A similar effective electron density can be found for the Finland radar when adjusted on variations of $\Theta_{F}$.

\section{OOFA convection estimates for the 12 February 1999 event}

Now we attempt to predict the magnitude and the azimuth of $\boldsymbol{V}_{\boldsymbol{E} \times \boldsymbol{B}}$ from the 1-o-s STARE velocities using the OOFA method. Figures $6 \mathrm{a}, \mathrm{b}$ show the EISCAT $\boldsymbol{V}_{\boldsymbol{E} \times \boldsymbol{B}}$ electron flow azimuth and magnitude (blue lines) and the stereoscopic STARE MP $\mathbf{V}_{p h}$ velocity estimates (green lines). We also show here the OOFA-predicted electron flow azimuth and magnitude with grey lines. By comparing the traces, one can conclude that the OOFA predictions correspond to the EISCAT $\boldsymbol{E} \times \boldsymbol{B}$ data reasonably well. Here we again mention the large difference in the EISCAT and STARE collecting areas (see Sect. 3). The latter can be a source of data distinction.

By comparing Fig. $3 b$ and Fig. $6 \mathrm{~b}$ one can see an important difference between the IAA and OOFA estimates: the IAA method failed to predict moderate and relatively high electron drifts of $\sim 1000 \mathrm{~m} / \mathrm{s}$ between 11:30 and 13:30 UT, while the OOFA method predicts them reasonably well. For later times, with the increase of the electron drifts to 1500 $2000 \mathrm{~m} / \mathrm{s}$, both methods give reasonable estimates. In terms of the $\boldsymbol{E} \times \boldsymbol{B}$ azimuth, OOFA predicts it reasonably well (Fig. 6a, grey line), while IAA predicts it with a $5-15^{\circ}$ counterclockwise offset with the respect to $\boldsymbol{V}_{\boldsymbol{E} \times \boldsymbol{B}}$ (Fig. 3a, brown line). Dotted grey lines in Figs. 6a, b show the so-called OOFA DP-predicted values, if one applies $h_{e f f}$ and $\Psi_{\text {eff }}$ to the original STARE DP velocities. These values are similar at the IAA predictions, where both the velocity underestimation and the azimuth offset are easily seen.

Again, as in paper 1, one can see that OOFA gives surprisingly good velocity estimates, but now for the evening event. One can certainly argue that the "agreement" resulted from a careful analysis of the event for which information on the density distribution was employed. To check how "robust" the OOFA model is and whether one can use the OOFA convection estimates directly, without the EISCAT support, we look at other events for which information on the $N(h)$ profiles is intentionally ignored. We consider two cases which 

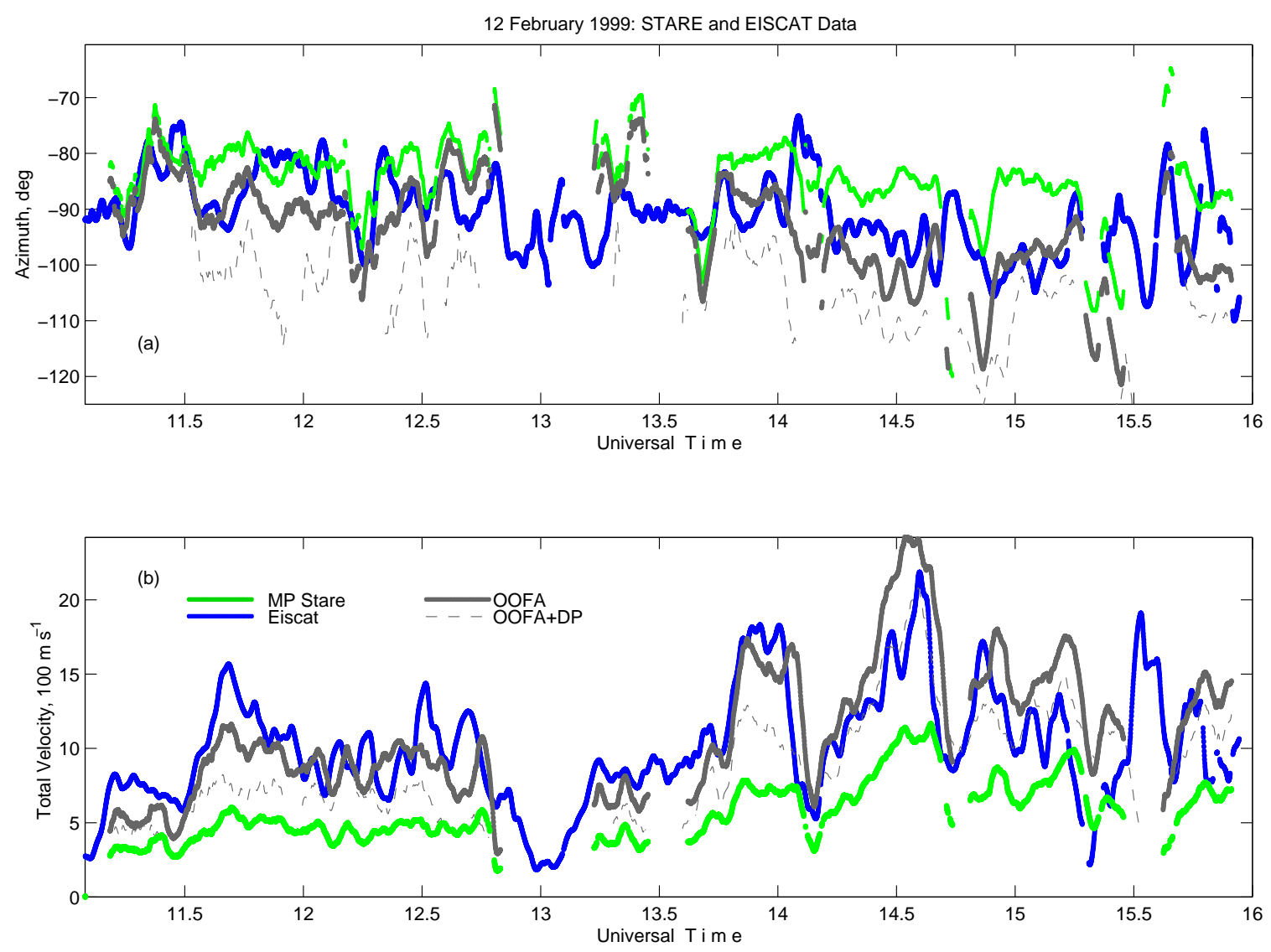

Fig. 6. The MP STARE and EISCAT parameters as in Fig. 3, together with the OOFA-predicted electron flow azimuth (panel (a)) and flow velocity (panel (b)), grey lines; dashed grey lines are the similar azimuth and velocity if the OOFA is applied to the STARE DP data.

have already been described by using $N(h)$ profiles but we will ignore this information now. In this way, we can assess the importance of information about $N(h)$ profiles. We also consider three additional events for which density profiles were never considered. Below we assume the mean STARE/EISCAT velocity ratio $\left|\boldsymbol{V}_{p h}\right| /\left|\boldsymbol{V}_{\boldsymbol{E} \times \boldsymbol{B}}\right|$ of 0.55 and the mean $\mathbf{V}_{p h}$ azimuth offset of $9^{\circ}$, as seen in Figs. 5d, e.

\section{OOFA convection estimates for additional events}

Figure 7 shows the results of non-EISCAT supported OOFA predictions for four evening events and for one morning event for which independent EISCAT convection data in CP$1 \mathrm{~K}$ mode were used for comparison. Although evening and morning events of 12 February 1999 were considered above and earlier in paper 1 , we included them in the analysis, to evaluate the OOFA predictions with the simplier approach taken in this section, where we ignore $N(h)$ data. We did not use electron density data intentionally for these events, contrary to the more sophisticated approach given above and in paper 1.

In all five narrow panels for the flow azimuth, one can see that the STARE MP merge azimuth, green line, is shifted from just several degrees to $\sim 15^{\circ}$ clockwise with respect to the EISCAT azimuth, blue line. Contrary to this, the OOFA (grey line) predicts the azimuth better and reasonably well. It is interesting that for the case of 12 February evening event (RHS bottom panels), the OOFA model with fixed azimuth offset (considered in the present section) predicts the electron flow azimuth better than the more detailed approach of Fig. 6a. We believe this occurs because the EISCAT $N(h)$ profiles cannot characterize properly the mean electron densities inside the STARE scattering volume, which is $\sim 3$ orders of magnitude larger than the EISCAT volume of measurements.

In the five large panels of Fig. 7, the EISCAT and STARE velocity magnitudes are compared (shown by blue and green lines, respectively). The grey line represents the OOFA predicted (with fixed azimuth offset model) electron flow magnitude. A reasonable agreement can be found for all events. Importantly, the flow strength was moderate and strong, thus covering the most difficult conditions for predictions. Also, the events were for different seasons.

\section{Discussions}

In this study we focused on joint STARE-EISCAT observations in the afternoon and early evening sector. 

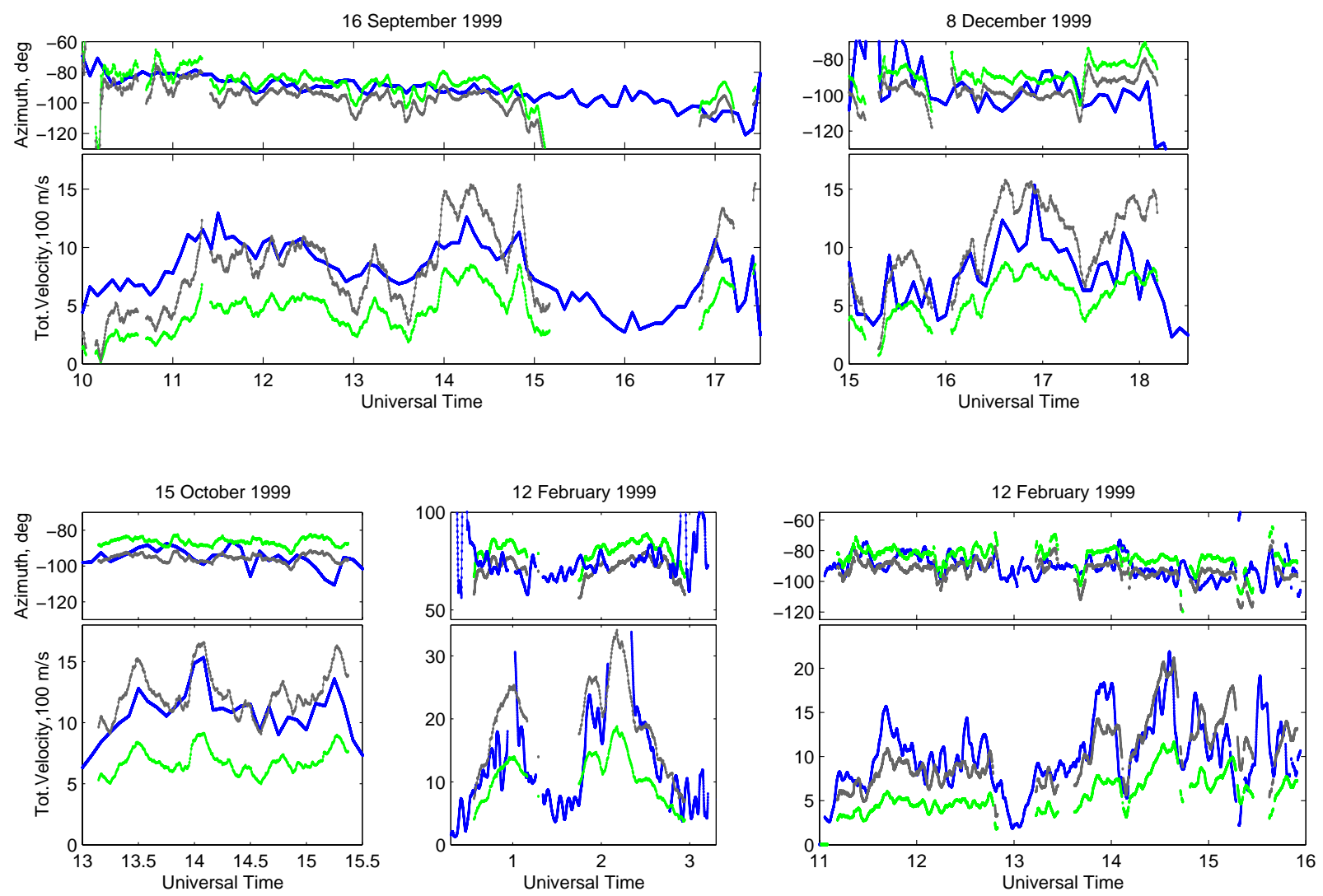

Fig. 7. Four evening and one morning case of the EISCAT electron flow azimuth (narrow panels) and magnitude (wider panels), blue lines, together with the OOFA estimates of the electron flow (grey lines) based on the STARE MP parameters (green lines).

7.1 Where was the area of STARE-EISCAT measurements?

The four evening cases (considered in Fig. 7) corresponded to observations at moderately disturbed conditions within a wide band of the afternoon-evening eastward electrojet (westward electron flow). The positive magnetic perturbations in the X-component over Scandinavia (as well as over Troms $\varnothing$ ) detected by the IMAGE magnetometers were 150-200 nT. $K_{p}$ indices were 3 on 8 December, $3+$ and $4+$ on 12 February, 4+, 3+ and 4- on 16 September, and 4- and 5 on 15 October. No all-sky camera measurements were available for all cases except on 8 December. In the latter case we saw that the EISCAT spot was inside the subauroral diffuse luminosity band, $1-2^{\circ}$ poleward of its equatorward edge and the main ionospheric trough.

An inspection of data collected by the Finland radar shows that all considered cases started near the time of the so-called F-region dusk scatter events (DUSE) first described by Ruohoniemi et al. (1988) and studied in more detail by Hosokawa et al. (2002). The EISCAT $\boldsymbol{E} \times \boldsymbol{B}$ drifts of $500-600 \mathrm{~m} / \mathrm{s}$ and the appearence of intense $1-\mathrm{m}$ irregularities seen by the STARE radars indicate the significance of the ambient poleward electric field in the generation of DUSE. We should note that a quick increase in the poleward-oriented E-field and the excitation of field-aligned irregularities can actually start even earlier, near the local noon, as on 16 September event.

In all our cases, we believe that after crossing the duskside end of the mid-latitude trough (Hosokawa et al., 2002) the EISCAT spot entered the subauroral diffuse luminosity belt and stayed there most of the observational time. This conclusion can be supported by the data presented in Fig. 4 of Hosokawa et al. (2002). For both their events A and $\mathrm{B}$, the DUSE was followed by a narrow echo band located $\sim 2^{\circ}$ equatorward of the EISCAT spot. A similar pattern of echo occurrence can be found in Uspensky et al. (2001), see their Fig. 3. These narrow echo bands are the E-region HF backscatter marking the equatorward edge of the diffuse luminosity belt produced by proton precipitations (Uspensky et al., 2001; Jayachandran et al., 2002). Incoherent scatter data show that the equatorward edge of the diffuse luminosity belt in the afternoon-evening is located roughly under the main ionospheric trough (e.g. Weber et al., 1985). We should say that although we believe that the EISCAT spot was mainly inside the diffuse luminosity belt, it could have briefly crossed an equatorward edge of the auroral oval. It might have happened during short electrojet intensifications seen by the IMAGE magnetometers at $\sim 14: 40$ UT on 12 February and $\sim$ 14:50 UT on 16 September 1999 (data are not shown here). 


\subsection{STARE-EISCAT comparison}

In this study we considered the relationship between the phase velocity of electrojet plasma waves seen by the STARE radars and the electron drift observed by EISCAT. This issue was the subject of a number of previous studies, e.g. Nielsen and Schlegel (1983, 1985), Reinleitner and Nielsen (1985), Kofman and Nielsen (1990), Kustov et al. (1990), Haldoupis and Schlegel (1990), Kustov and Haldoupis (1992), Haldoupis et al. (1993), Chen et al. (1995), Nielsen et al. (2002), Koustov et al. (2002) and Uspensky et al. (2003), (paper 1). Observations from two different directions with respect to the electron flow were distinguished in these studies. For large flow angles it was assumed by most researchers that the observed STARE velocity is the cosine component of the $\boldsymbol{E} \times \boldsymbol{B}$ electron drift (Nielsen and Schlegel, 1983, 1985; Nielsen et al., 2002). However, some previous studies indicated violation of this rule (e.g. Kustov et al., 1990, and paper 1).

For observations along the electron flow, the basic assumption was that the cosine rule is not valid and the 1-o-s velocity is "saturated" around the local ion-acoustic speed (e.g. Nielsen and Schlegel, 1983; 1985). As a result, the standard merged STARE data reduction scheme brings errors in the STARE flow angles down to a few tens of degrees (Robinson, 1993). Similar 5-10 jumps in the STARE IAA flow azimuth can be seen in Fig. 3a, 13:40-15:30 UT (thin brown line), when the Norway radar detects $>\sim 300 \mathrm{~m} / \mathrm{s}$ phase velocities. In contrast to IAA, OOFA does not modify the cosine rule and the standard merging does not imply additional errors.

In spite of the generally accepted opinion that the irregularity phase velocity of electrojet irregularities along the electron flow is limited to the ion-acoustic speed (e.g. Chen et al., 1995; Robinson, 1986; Robinson and Honary, 1990; see references therein), the issue is not as simple as it sounds (Janhunen, 1994; Oppenheim et al., 1996, Foster, 2002). Haldoupis and Schlegel (1990) studied the relationship between the STARE 1-o-s velocity inside the FB instability cone and the ion-acoustic speed $C_{s}$ obtained from concurrent EISCAT measurements. They indicated that there is no clear trend in the $\mathbf{V}_{p h}$ and $C_{s}$ relationship, although they pointed out that the phase velocity is often limited to values in the vicinity of $C_{s}$. In the westward electrojet they found the mean $\mathbf{V}_{p h}$ to be about 30-40\% larger than $C_{s}$, in the eastward electrojet about $20 \%$ smaller than $C_{s}$ (see their Fig. 8). To use their conclusions one has to note that the authors cut off all points where velocities were less than $280 \mathrm{~m} / \mathrm{s}$. (If we make a plot of $\mathbf{V}_{p h}$ versus $C_{s}$ for our data in Fig. 2, where electron velocities are equal to or greater than $C_{s}$, we obtain quite a few points for which $\mathbf{V}_{p h}$ is even half the value of $C_{s}$ ). Haldoupis et al. (1993) found that the merged irregularity phase velocity during electron heating usually increased, but in one strong heating event, the irregularity speed remained almost independent of the very large electron temperatures. Opposite examples with the phase velocity enhancements without any heating are also common in their data.
In this study (also in paper 1) we presented the data that support the OOFA approach. The evidence is the estimates of the STARE velocity reduction (due to signal reception from all electrojet heights) as compared to the $\boldsymbol{E} \times \boldsymbol{B}$ electron flow component. For typical values of reduction, 0.55 , one can easily see that both the Finland and Norway velocities match the EISCAT components quite well for most of the time, Fig. 2. As a result, the implication of the performed OOFA analysis (where we intentionally ignored EISCAT $N(h)$ data) is that one can make a quick correction of the STARE MP velocity estimates to obtain plasma convection even without the EISCAT support. It can be done by simply increasing the l-o-s velocities by a factor of 1.8 and by making about a $10^{\circ}$ anti-clockwise rotation of the velocity obtained from a simple merging of the MP 1-o-s velocities. The coefficient of 1.8 is somewhat larger than previously recommended by Kustov et al. (1989) (1.4). We believe that the update in the value of the depression coefficient reflects the change in the pulse sequencing in the STARE radars introduced in 1997. We should also warn that the above correction is applicable to the EISCAT spot crossing. Since aspect angles change across the STARE field of view, correction coefficients at other points need to be worked out.

Despite the reasonable agreement between the evening STARE phase velocities in the OOFA reduction scheme and the EISCAT electron flow velocities in different observational conditions, we are left with the impression that there still exist other linear and nonlinear effects that cannot be covered and/or explained by OOFA and are open for further study.

\subsection{Double-pulse velocities}

We confirmed in this study that the DP technique that is currently in use in the STARE routine data handling typically underestimates the phase velocity of echoes that are measured by the MP technique. Similar effects were discussed earlier by Schlegel et al. (1986), Schlegel and Thomas (1988) and Whitehead (1988). The effect seems to be pronounced in the afternoon-evening sector when the Finland radar observes at large flow angles. In contrast, the Finland radar did not observe the DP velocity underestimates in the morning (for the same day) when the flow angles were $75-80^{\circ}$ (these data were used in paper 1).

We believe that there are two factors that contribute to the observed DP and MP velocity differences: The plasma physical properties of large aspect and flow angle waves, as hypothesized by Koustov et al. (2002) as well as in paper 1, and the specifics of the radar operation method. Indeed, in the DP scheme, the range ambiguity allows for an auroral clutter reception, which is only $30 \mathrm{~km}$ farther and $30 \mathrm{~km}$ closer than the radar target bin. This is a result of the $200-\mu$ s DP separation. The ideal case with no clutter influence is when there is no correlation between the clutter and target pulses inside the 20 -s integration time. Perhaps this condition is too difficult to satisfy for some specific cases. One may wonder why the velocity underestimation is stronger for the evening sector 
observations. We believe the reason is that evening echoes are more diffuse and homogenous in their nature, so that the spatial coherence of echoes from spatially different areas is more significant in the evening than in the morning. Morning echoes are well known to be often discrete and patchy. These echo features are well described in early publications on auroral radar backscatter (e.g. by Unwin (1966)). Another feature is the behaviour of the echo spectral width and asymmetry (Haldoupis et al., 1984; Schlegel et al., 1986). Our hypothesis can be checked by looking at the double-pulse and multi-pulse STARE measurements. This work is currently under way and preliminary data show that the doublepulse velocities in the afternoon-evening are systematically smaller than the multi-pulse ACF-fit velocities. A more detailed analysis will be the subject of a forthcoming paper.

By means of the MP technique we also found that the STARE MP merge flow azimuth was shifted systematically clockwise by $5-15^{\circ}$ with respect to the EISCAT electron drift (see Figs. 6 and 7). This effect is less pronounced or even absent if one uses the STARE DP velocities (Fig. 3a). However, moderate azimuth clockwise offsets can be recognized in the STARE/EISCAT data of Nielsen and Schlegel (1985) for the electron drifts of more than $500 \mathrm{~m} / \mathrm{s}$ (see their Fig. 4). A similar tendency can be seen in the data of the recent paper by Nielsen et al. (2002) (their Fig. 5). This feature can be explained by the ion velocity contribution to the irregularity phase velocity, as first discussed by Uspensky et al. (2003), (paper 1). The ion velocity contribution can also be seen in the present case after $\sim 14: 00$ UT and around $\sim 15: 00$ UT when the Finland MP velocity (Fig. 2b) is close or shortly higher than its EISCAT plasma flow component. Note that this happens when the EISCAT electron flow differs only 10$15^{\circ}$ from the normal to the Finland antenna beam 4 , just as it was predicted in paper 1 .

\subsection{Low velocities}

We should note that according to Nielsen et al. (2002) "for large flow angles the Doppler shifts are equal to the component of the electron flow velocity on the line of sight". (A similar conclusion was made by Nielsen and Schlegel, 1983; 1985; Kofman and Nielsen, 1990). We feel that this is an overstatement of the actually presented data, at least for some periods. For example, between 11:20-13:40 UT in our Fig. 2, the Norway STARE velocity was $\sim 250 \mathrm{~m} / \mathrm{s}$, roughly two times smaller than both the $\boldsymbol{E} \times \boldsymbol{B}$ electron flow component and the $105-111 \mathrm{~km}$ isothermal ion-acoustic speed. The Norway STARE flow angle during this time was $\sim 60^{\circ}$ so that observations were performed outside the instability cone. Such data, on the other hand, can be explained by the OOFA model, since it predicts $\left|\boldsymbol{V}_{p h}\right| /\left|\boldsymbol{V}_{\boldsymbol{E} \times \boldsymbol{B}}\right|$ ratios to be somewhere between $0.45-0.65$ (see, e.g. Fig. 5e).

The OOFA method of STARE convection estimates is based on the quantitative two-fluid description of the irregularity velocity. However, it does not invalidate the IAA method; our analysis showed a reasonable performance of IAA most of the time when the phase velocities were in- creased. For certain periods, the OOFA method was superior over IAA, but certainly more work is needed to assess why and when this happens. As far as the IAA scheme is concerned, we see some internal problems within it. For the event considered, the IAA model proposed to use a critical value (threshold for the irregularity excitation) slightly less than $300 \mathrm{~m} / \mathrm{s}$; however, such a value seems to be too small when compared to the actual EISCAT ion-acoustic velocities of up to $500-600 \mathrm{~m} / \mathrm{s}$. If one assumes larger thresholds, the IAA predictions worsen.

Nielsen and Schlegel (1985) and also recently, Nielsen et al. (2002) noted the existence of low 1-o-s velocities, which they explained as the non-two-stream waves, or recently, as a result of the DP method of measurements (Nielsen et al., 2002). The low velocities are poorly handled by the IAA scheme while there is no problem within the OOFA scheme.

\subsection{The errors of OOFA predictions}

It is important to establish how accurate the OOFA electron flow predictions are on a more significant database. This work is in our plans. It is desirable to encompass a variety of electric field magnitudes and directions. Of special interest is the situation when the electric field is near the threshold for the FB plasma instability because the empirical IAA recipes are slightly different for westward and eastward electrojets while the physics involved is the same.

With the data analyzed so far, natural explanation for the discovered EISCAT and STARE inconsistencies can be the very different spatal resolutions of the instruments whose collecting areas are 2-3 orders of magnitude different. One can appreciate the importance of this factor by comparing OOFA predictions for the case of utilization of the electron density information, Fig. 6, and for the case of ignoring it, Fig. 7, right-hand side, bottom panels. One can see better OOFA azimuth predictions in Fig. 7 and the difference between velocity magnitudes, particularly around $\sim$ 14:00 UT. Similar OOFA predictions for the morning case show a neglegible difference (see middle bottom panels in Fig. 7 and paper 1, their Fig. 7). An example of a local effect in EISCAT data can be seen, perhaps, at $\sim 15: 35$ UT. The EISCAT detected the short convection enhancement which was nearly simultaneous with a drop in STARE SNRs but was not detected in the magnetometer and electron density data (see Fig. 2, panels (c) and (a), respectively; magnetometer data are not shown).

Comparison of Fig. 6 and the EISCAT data, Fig. 4, panel (c), shows a tendency for a weak velocity overestimation at $\sim$ 14:30 and 14:55 UT within two intervals, with increased electron drifts and increased ion temperatures. (The increased ion temperature at $\sim 13: 55$ UT does not create a velocity overestimation). Similar weak tendency of a velocity overestimation can be seen at 17:00 UT on 8 December, at $\sim 14: 10$ UT on 16 September, and $\sim 13: 30$ and $\sim 15: 20$ UT on 15 October 1999 (the temperature data not shown here). It seems this is in accordance with the expectation that a stronger electron flow stimulates more turbulent conditions 
with stronger nonlinear effects and enhanced electron collisions. Then the weak velocity overestimation could result from our assumption of electric field independent collision frequencies.

One more factor of OOFA velocity overestimation can be the underestimation of the backscatter anisotropy factor $a$ $(\sim 50)$ used in modelling. The choice of $a$ was based on the experimental data for the aspect sensitivity of echoes, $10 \mathrm{~dB} /{ }^{\circ}$ (Chesnut, 1968). Measurements with better antenna resolution (e.g. Foster et al., 1992) indicate that the aspect sensitivity can be stronger at $\sim 15 \mathrm{~dB} /{ }^{\circ}$. This would lead to the factor $a \sim 60^{\circ}$.

\section{Conclusions}

The main conclusions of the present study can be summarized as follows:

1. We showed that the double-pulse technique that is currently in use in the STARE routine data handling typically underestimates the true phase velocity as measured by the multi-pulse technique. The effect seems to be more pronounced for the Finland radar observations at large flow angles, especially in the afternoon and evening. It seems that a future data reduction scheme should include both MP and DP data sets.

2. We demonstrated that stereoscopic merging of Finland and Norway MP velocities still gives convection underestimation. It was found that the clockwise $\sim 10^{\circ}$ azimuth offset of the STARE flow velocity vector with respect to the EISCAT electron flow vector is seen well in the afternoon and evening, in agreement with the conclusions of paper 1 , where the effect was reported for the morning observations.

3. The considered OOFA concept of STARE echo formation suggests that the difference between the STARE MP line-of-sight velocities and EISCAT electron drifts along STARE beams, especially evident for fast electron flows, can be, to a large extent, a product of a moderate, effective backscatter off-orthogonality. The reason is that for any radar cell, the auroral echoes are collected from various heights with perfect orthogonality at only one of them. Echo components coming from above and below the height with perfect orthogonality are received at non-zero aspect angles (up to $1-1.5^{\circ}$ ), and their velocity is slower than the $\boldsymbol{E} \times \boldsymbol{B}$ plasma drift component.

4. The effective aspect angle and the effective backscatter altitude combined with the plasma fluid equation for the irregularity velocity allow one to predict, with reasonable accuracy, the electron flow velocity (as measured by EISCAT) in the afternoon and evening for moderate and enhanced velocity magnitudes, as demonstrated for five different events ( $22 \mathrm{~h}$ in total).
5. The OOFA-based scheme of STARE electron flow predictions involves only two correcting factors, the azimuth offset $\left(\sim 10^{\circ}\right)$ and the line-of-sight velocity enhancement coefficient (a factor of $\sim 1.8$ ). When OOFA is applied, STARE predicitons of the flow direction are improved as compared to simple MP velocity merging.

6. We compared the OOFA and IAA convection predictions for one event and found the OOFA predictions to be superior for moderate electon flows and of comparable quality for fast electron flows.

Acknowledgements. This research has been supported by the Academy of Finland provided to M. U. and by an NSERC Research Grant to A. K. The authors thank G. Leppelmeier, for useful discussion and comments. EISCAT is funded collaboratively by the research councils of Finland, France, Germany, Japan, Norway, Sweden and UK funding body, PPARC. STARE is operated by the MaxPlanck-Institut für Aeronomie, Germany, and by the Finnish Meteorological Institute, Helsinki, Finland, in cooperation with Technical University of Trondheim, Norway. Our sincere thanks go to D. Farley, Cornell University, for valuable comments and discussions. We are greatful to A. Kozlovsky for his kind help in the data handling.

Topical Editor M. Lester thanks M. Kosch and K. Hosokawa for their help in evaluating this paper.

\section{References}

Andre, D.: The dependence of the relative backscatter cross section of $1 \mathrm{~m}$ density fluctuations in the auroral electrojet on the angle between electron drift and radar wave vector, J. Geophys. Res., 88, 8043-8049, 1983.

Baumjohann, W., and Opgenoorth, H. J.: Electric fields and currents associated with active aurora, in Magnetospheric Currents, edited by Potemra, T. A., 77-85, AGU, Washington, D.C., 1984.

Chen, P-R., Yi, L., and Nielsen, E.: Variations of the mean phase velocity of $1 \mathrm{~m}$ ionospheric plasma waves with plasma electron temperature, J. Geophys. Res., 100, 1647-1652, 1995.

Chesnut W.: Low frequency waves and irregularities in the auroral ionosphere as determined by radar measurements. Low frequency waves and irregularities in the ionosphere, edited by D'Angelo, N. and Reidel, D., 173-191, 1968.

Farley, D. T., Ierkic, H. M., and Fejer B. G.: The absolute scattering cross section at $50 \mathrm{MHz}$ of equatorial electrojet irregularities, J. Geophys. Res., 86, 1569-1575, 1981.

Farley D. T., and Providakes J.: The variation with Te and Ti of the velocity of unstable ionospheric two-stream waves, J. Geophys. Res., 94, 15 415-15 420, 1989.

Fejer, B. G., and Kelley, M. C.: Ionospheric irregularities, Rev. Geophys. Space Phys., 18, 401-454, 1980.

Foster, J. C., Tetenbaum, D., del Pozo, C. F., St-Maurice, J.-P., and Moorcroft, D. R.: Aspect angle variations in intensity, phase velocity and altitude for high-latitude $34 \mathrm{~cm}$ E-region irregularities, J. Geophys. Res., 97, 8601-8617, 1992.

Foster, J. C.: Millstone Hill E-region coherent scatter experiments: New perspectives on the sub-auroral electric field and its variability, Geophysical Institute, University of Alaska Fairbanks, Proceedings from SuperDARN, Valdez, AK, 2002.

Greenwald, R. A., Weiss, W., Nielsen, E., and Thomson, N. R.: STARE: A new radar auroral backscatter experiment in Northern Scandinavia, Radio Sci., 13, 1021-1029, 1978. 
Greenwald, R. A., Baker, K. B., Dudeney, J. R., Pinnock, M., Jones, T. B., Thomas, E. C., Villain, J.-P., Cerisier, J.-C., Senior, C., Hanuise, C., Hunsuker, R. D., Sofko, G., Koehler, J., Nielsen, E., Pellinen, R., Walker, A. D. M., Sato, N., and Yamagishi, H.: DARN/SuperDARN: A global view of the dynamics of highlatitude convection, Space Sci. Rev., 71, 761-796, 1995.

Haldoupis, C., Nielsen, E., and Ierkic, H. M.: STARE Doppler spectral studies of westward electrojet radar aurora, Planet. Space. Sci., 32, 1291-1300, 1984.

Haldoupis C. and Schlegel, K.: Direct comparison of $1 \mathrm{~m}$ irregularity phase velocity and ion-acoustic speeds in the auroral E-region ionosphere, J. Geophys. Res., 95, 18 989-19000, 1990.

Haldoupis, C., Schlegel, K., and Nielsen, E.: Some observations of radio auroral backscatter at $140 \mathrm{MHz}$ during E-region electron gas heating, Ann. Geophysicae, 11, 283-295, 1993.

Hanuise, C., Villain, J.-P., Gresillon, D., Cabrit, B., Greenwald, R. A., and Baker, K. B.: Interpretation of HF radar ionospheric Doppler spectra by collective wave scattering theory, Ann. Geophysicae, 11, 29-39, 1993.

Hosokawa, K., Sugino, M., Lester, M., Sato, N., Yukimatu, A. S., and Iyemori, T.: Simultaneous measurement of duskside subauroral irregularities from the CUTLASS Finalnd radar and EISCAT UHF system, J. Geophys. Res., 107, A12, 1457, doi:10.1029/2002JA009416, 2002.

Huuskonen, A.: High resolution observations of the collision frequency and temperatures with the EISCAT radar, Planet. Space Sci., 37, 211-221, 1989.

Inhester, B., Baumjohann, W., Greenwald, R. A., and Nielsen, E.: Joint two-dimensional observations of ground magnetic disturbances and ionospheric electric fields associated with auroral zone currents: 3. Auroral zone currents during passage of a westward travelling surge, J. Geophys. Res., 49, 155-162, 1981.

Janhunen, P.: Perpendicular particle simulation of the E-region Farley-Buneman instability, J. Geophys. Res., 99, 11461$11473,1994$.

Jayachandran, P. T., Donovan, E. F., MacDougal, J. W., Moorkroft, D. R., St. Maurice, J.-P., and Prikryl, P.: SuperDARN E-region backscatter boundary in the dusk-midnight sectortracer of equatorward boundary of auroral oval, Ann. Geophysicae, 20, 1899 1904, 2002.

Kofman W., and Nielsen, E.: STARE and EISCAT measurements: Evidence for the limitation of STARE Doppler velocity observations by the ion acoustic velocity, J. Geophys. Res., 95, 19131$19136,1990$.

Kosch, M. J. and Nielsen, E.: Statistical average estimates of high latitude field-aligned currents from the STARE and SABRE coherent VHF radar systems, Adv. Space Res., 27, 1239-1244, 2001.

Kustov, A. V., Uspensky, M. V., Huuskonen, A., Kangas, J., Nielsen, E., and Fejer, B. G.: On the influence of altitudinal profile of auroral backscatter on electric field measurements in the STARE experiment, In: Instabilities and wave phenomena in the ionosphere-thermosphere system, Gorky (USSR), 205-209, 1989.

Kustov, A. V., Uspensky, M. V., Huuskonen, A., Kangas, J., and Nielsen, E.: Effect of the altitude profile of auroral scattering on electric field measurements in the STARE experiment, Geom. and Aeronomie, (English), 30, 384-388, 1990.

Kustov, A. V. and Haldoupis, C.: Irregularity drift velocity estimates in radar auroral backscatter, J. Atmos. Terr. Phys., 1992, 54, 415-423, 1992.

Koustov, A. V., Danskin, D. W., Uspensky, M. V., Ogawa, T., Jan- hunen, P., Nishitani, N., Nozawa, S., Lester, M., and Milan, S.: Velocities of auroral coherent echoes at 12 and $144 \mathrm{MHz}$, Ann. Geophysicae, 20, 1647-1661, 2002.

Nielsen, E. and Schlegel, K.: A first comparison of STARE and EISCAT electron drift velocity measurements, J. Geophys. Res., 88, 5745-5750, 1983.

Nielsen, E. and Schlegel, K.: Coherent radar Doppler measurements and their relationship to the ionospheric electron drift velocity, J. Geophys. Res, 90, 3498-3504, 1985.

Nielsen, E., Uspensky, M., Kustov, A., Huuskonen, A., and Kangas, J.: On the dependence of the Farley-Buneman turbulence level on ionospheric electric field, J. Atmos. Terr. Phys., 50, 601-605, 1988.

Nielsen, E.: Coherent radar technique, World Ionosphere/Thermosphere Study, WITS Handbook, 2, eds. C.H. Liu, ICSU/SCOSTEP, U of Illinois, 1989.

Nielsen, E.,Bruns, M., Pardowitz, I., Bemmann, L., Janhunen, P., and A. Huuskonen, STARE: Observations of a field-aligned current, Geophys. Res. Lett., 26, 1, 21-24, 1999.

Nielsen, E., del Pozo, C. F., and Williams, P. J. S.: VHF coherent radar signals from the $\mathrm{E}$ region ionosphere and the relationship to electron drift velocity and ion-acoustic velocity, J. Geophys. Res., 107, (A1), 10.1029/2001JA900111, 2002.

Nielsen, E. and Rietveld, M. T.: Observations of backscatter autocorrelation functions from 1.07 meter ionospheric irregularities generated by the EISCAT Heater Facility, J. Geophys. Res., J. Geophys. Res., 108, (A5), 1029/2002JA009537, 2003.

Oksman, J., Uspensky, M. V., Starkov, G. V., Stepanov, G. S., and Vallinkoski, M.: The mean fractional electron density fluctuation amplitude derived from auroral backscatter data, J. Atmos. Terr. Phys., 48, 107-113, 1986.

Oppenheim, M., Otani, and N. Ronchi, C.: Saturation of the FarleyBuneman instability via nonlinear electron $\boldsymbol{E} \times \boldsymbol{B}$ drifts, J. Geophys. Res., 101, 17 273-17 286, 1996.

Pfaff, R. F., Kelley, M. C., Fejer, B. G., Kudeki, E., Carlson, C. W., Pedersen, A., and Hausler, B.: Electric field and plasma density measurements in the auroral electrojet, J. Geophys. Res., 89, 236-244, 1984.

Reinleitner, L. A. and Nielsen, E.: Self-consistent analysis of electron drift velocity measurements with the STARE/SABRE system, J. Geophys. Res., 90, 8477-8486, 1985.

Robinson, T. R.: Towards a self-consistent nonlinear theory of radar aurora backscatter, J. Atmos. Terr. Phys., 48, 417-422, 1986.

Robinson, T.,R., and Honary, F.: A resonance broadening kinetic theory of the modified-two-stream instability: Implications for radar auroral backscatter experiments, J. Geophys. Res., 95, 1073-1085, 1990.

Robinson, T.: Simulation of convection flow estimation error in VHF bistatic auroral radar systems, J. Atmos. Terr. Phys., 11, 1033-1050, 1993.

Ruohoniemi, J. M., Greenwald, R. A., Villain, J.-P., Baker, K. B., Newell, P. T., and Meng, C.-I.: Coherent HF radar backscatter from small-scale irregularities in the dusk sector of the subauroral ionosphere, J. Geophys. Res., 93, 12 871-12 882, 1988.

Schlegel, K.: Interpretation of auroral radar experiments using a kinetic theory of the two-stream instability, Radio Sci., 18, 108$118,1983$.

Schlegel, K., Thomas, E. C., and Ridge, D.: A statistical study of auroral radar spectra obtained with SABRE, J. Geophys. Res., 91, 13 483-13 492, 1986.

Schlegel, K., and Thomas, E.C.: Reply, J. Geophys. Res., 93, 5987, 1988. 
Uspensky, M. V.: On the altitudinal profile of auroral radar backscatter, Radio Sci., 20, 735-739, 1985.

Uspensky, M., Eglitis, P., Opgenoorth, H., Starkov, G., Pulkkinen, T., and Pellinen, R.: On auroral dynamics observed by HF radar: 1. Equatorward edge of the afternoon- evening diffuse luminosity belt, Ann. Geophysicae, 18, 1560-1575, 2001.

Uspensky, M., Koustov, A., Janhunen, P., Pellinen, R., Danskin, D., and Nozawa, S.: STARE velocities: Importance of offorthogonality and ion motion, Ann. Geophysicae, 21, 729-744, 2003.
Unwin, R. S.: The morphology of the VHF radio aurora at sunspot maximum -II. The behaviour of different echo types, J. Atmos. Terr. Phys., 28, 1183-1194, 1966.

Weber, E. J., Tsunoda, R. T., Buchau, J., Sheehan, R. E., Strickland, D. J., Whiting, W., and Moore, J. G.: Coordinated measurements of auroral zone plasma enhancements, J. Geophys. Res., 90, 6497-6513, 1985.

Whitehead, J. D.: Comment on "Statistical study of auroral radar spectra obtained with SABRE" by Schlegel, K., Thomas, E. C., and Ridge, D., J. Geophys. Res., 93, 5985-5986, 1988. 